\title{
$\mathrm{Cu}(\mathrm{I})$ 催化的不对称直接插炔 Aldol 反应构建手性 2,3-联烯醇
}

\author{
手光尧陈应春* \\ (四川大学华西药学院 成都 610041)
}

\section{Construction of Chiral 2,3-Allenols through Copper(I)-Catalyzed Asymmetric Direct Alkynylogous Aldol Reaction}

\author{
Ran, Guangyao Chen, Yingchun* \\ (West China School of Pharmacy, Sichuan University, Chengdu 610041)
}

手性联烯是一类具有丙二烯结构的轴手性化合物, 不仅广泛存在于众多天然产物 ${ }^{[1]}$ 、药理活性分子及功能 材料中 ${ }^{[2]}$, 还是有机化学中非常重要的合成砌块 ${ }^{[3]}$. 其 中, 含有一个中心手性和轴手性的 2,3-联烯醇结构是类 胡萝卜素类、萜类以及溴代联烯天然产物的核心片段, 表现出广泛的生理活性, 并且由于同时含有联烯和醇羟 基官能团, 也具有多样的反应化学. 因此, 2,3-联烯醇的 不对称合成具有重要的研究意义.

从炔基取代的烯醇硅梄或联烯酸酯出发, 通过形成 炔基烯醇负离子从而与酫或酮发生不对称插炔 aldol 反 应, 是一种高效合成手性 2,3-联烯醇的策略(Scheme 1). 2016 年, List 小组 ${ }^{[4]}$ 采用手性磺酰亚胺(disulfonimide) 实 现了首例芳香醛的 Mukaiyama 类型的不对称插炔 aldol 反应; 冯小明和刘小华团队 ${ }^{[5]}$ 利用 $\mathrm{Au}$ /手性氮氧配体实 现了联烯酸酯与靛红的直接不对称插炔 aldol 反应. 然 而上述例子均未能实现脂肪醛的相关转化, 可能底物易 发生自身缩合等副反应. 2018 年, Yu 和 Kim 团队 ${ }^{[6]}$ 利用 化学计量的手性硼试剂, 预先与联烯酸酯形成手性炔基 烯醇嗍中间体, 随后进攻多种醛化合物形成相应的 2,3联烯醇. 尽管该方法能实现脂肪醛的有效转化, 但采用 化学计量手性磀试剂严重影响了反应的经济性.

中国科学院上海有机化学研究所殷亮课题组一直 致力于 $\mathrm{Cu}(\mathrm{I})$ 催化的不对称插烯加成反应研究, 系统发 展了不对称直接插烯 aldol 反应 ${ }^{[7]}$ 、Mannich 型反应 ${ }^{[8]}$ 等. 最近, 他们推测 $\mathrm{Cu}(\mathrm{I})$ 能与非共轭炔酸酯生成亲核性铜 物种, 进而与醛类化合物发生不对称加成反应(Scheme $2)^{[9]}$. 经系统篎选后, 该小组发现芳(杂)醛 1 与 $\beta$-炔酸酯 2 在 $\mathrm{Cu}\left(\mathrm{CH}_{3} \mathrm{CN}\right)_{4} \mathrm{PF}_{6}$ 和大位阻双膦配体 $(R)$-DTBM-SEG-
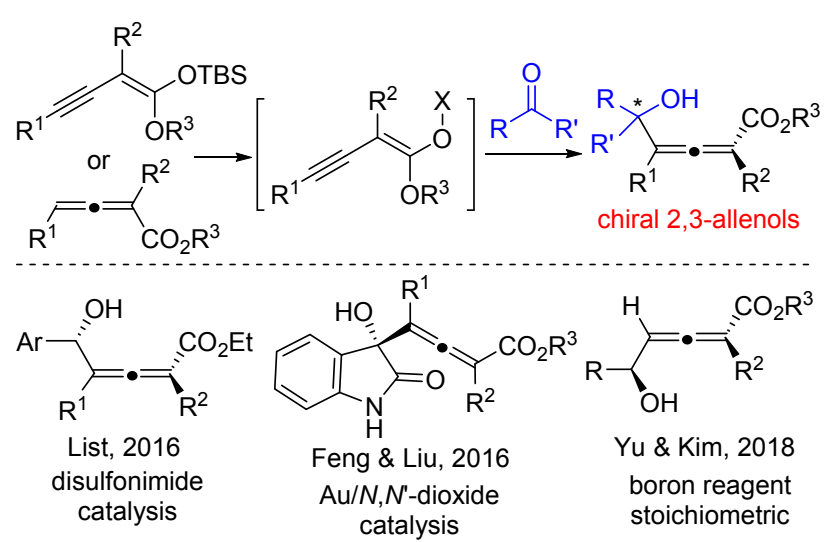

图式 1 不对称插炔 aldol 反应构建手性 2,3-联烯醇 Scheme 1 Construction of chiral 2,3-allenols via asymmetric alkynylogous aldol reaction

PHOS (L1)的催化以及 2-叔丁基四甲基胍为碱(Barton's Base)的条件下, 能顺利地进行插炔 aldol 反应, 以良好 的收率、优异的 $\gamma$-区域选择性以及立体选择性得到相应 的手性 2,3-联烯醇(3) (Scheme 2). 更为重要的是, 更具 挑战性的脂肪醛 4 (包括链状及环状), 也可与 $\beta$-炔酸丁 炔酯(5)在 2,4,6-三甲苯基铜 [mesitylcopper(I)]和手性膦 配体 $(R, R)-\mathrm{Ph}-\mathrm{BPE}(\mathbf{L} 2)$ 的作用下, 以较好的收率、非对 映选择性和优异的对映选择性实现类似反应，多类官能 团均能良好兼容(Scheme 2).

他们还探索了该不对称直接插炔 aldol 反应的合成 应用性：该催化反应放大至克级，也能以优异结果得到 产物 3a(图 1); 另外, 含甾体结构芳香醛也可应用于该 策略，高效、高立体选择性地构建了含有表雄酮、二氢 胆固醇或炔雌醇骨架的 2,3-联烯醇(7 9), 为活性分子

* Corresponding author. E-mail: ycchen@scu.edu.cn. Published online February 25, 2020. 


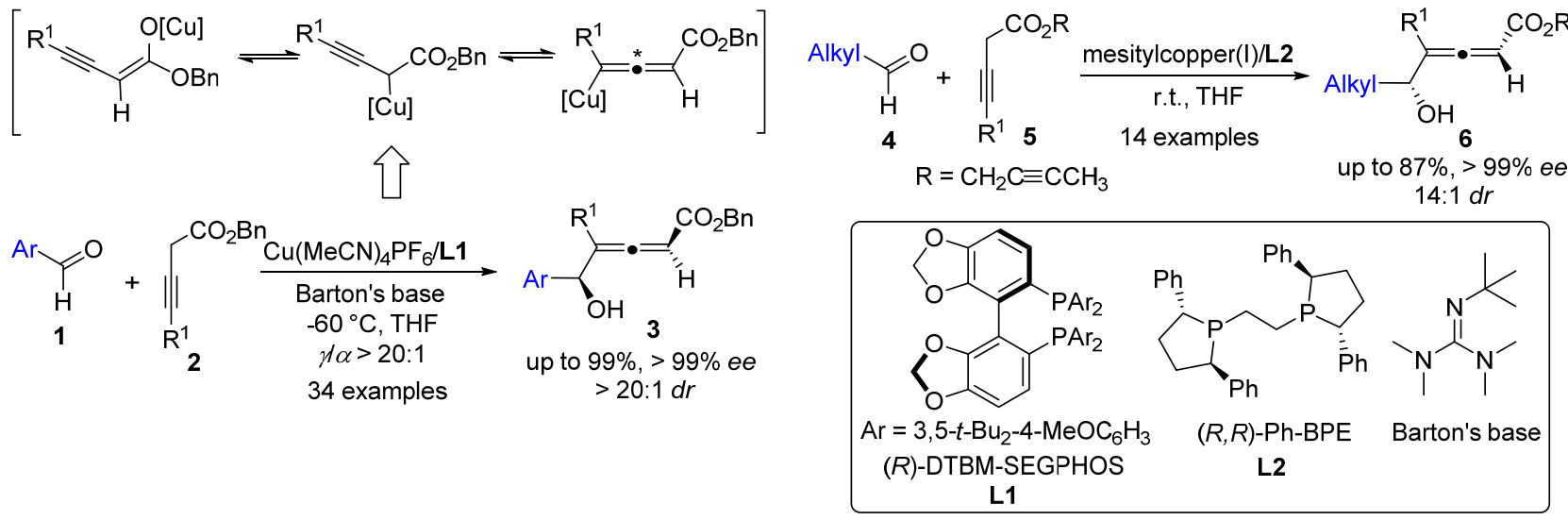

图式 $2 \mathrm{Cu}(\mathrm{I})$ 催化多种醛参与的不对称直接插炔 aldol 反应

Scheme $2 \mathrm{Cu}(\mathrm{I})$ catalyzed asymmetric direct alkynylogous aldol reaction with various aldehydes

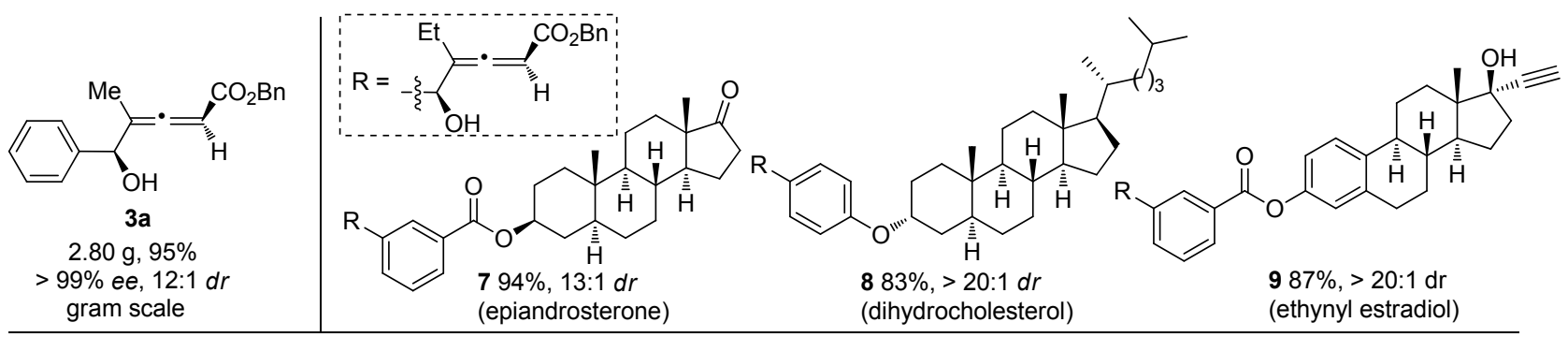

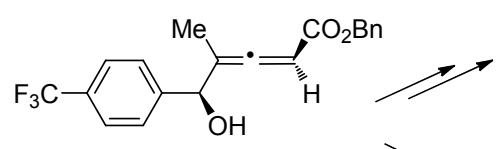

3b $99 \%$ ee, $>20: 1 d r$

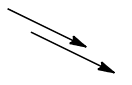

$\mathrm{H}$<smiles>CCCC(C)C</smiles>

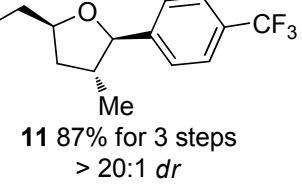

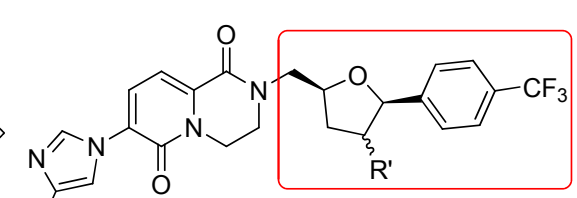

$\mathrm{AR}^{\prime}=\alpha-\mathrm{Me}, \mathrm{IC}_{50}=38.4 \mathrm{nmol} / \mathrm{L}$ B R' $=\beta$-Me, $\mathrm{IC}_{50}=73.5 \mathrm{nmol} / \mathrm{L}$ active for Alzheimer's disease \& Down's syndrome

图 1 不对称插炔 aldol 反应的应用

Figure 1 Application of the asymmetric alkynylogous aldol reaction

的快速衍生化提供更多选择. 同时, 作者还从插炔 aldol 产物 3b 出发, 通过几步简单反应快速合成了多取代呋 喃的一对差向异构体 10 和 11, 是有望用于治疗多种神 经性疾病的化合物的关键片段.

综上所述，殷亮小组采用 $\mathrm{Cu}(\mathrm{I})$ 与手性膦配体催化 体系成功实现了非共轭炔酸酯的不对称直接插炔 aldol 反应, 多种芳香醛甚至脂肪醛都能很好适用, 最终以良 好的收率和优异的区域、立体选择性地快速合成了一系 列手性 2,3-联烯醇化合物, 并能进一步转化得到多类具 有潜在应用价值的杂环化合物. 该方法学在手性联烯醇 的合成上展现出非常普适、高效、原子经济的优势, 有 望进一步拓展到更多的不对称反应, 并在天然产物、药 物合成等领域得到重要应用.

\section{References}

[1] Hoffmann-Röder, A.; Krause, N. Angew. Chem., Int. Ed. 2004, 43, 1196.

[2] Rivera-Fuentes, P.; Diederich, F. Angew. Chem., Int. Ed. 2012, 51, 2818.

[3] Yu, S.; Ma, S. Angew. Chem., Int. Ed. 2012, 51, 3074.

[4] Tap, A.; Blond, A.; N. Wakchaure, V.; List, B. Angew. Chem., Int. Ed. 2016, 55, 8962.

[5] Wang, G.; Liu, X.; Chen, Y.; Yang, J.; Li, J.; Lin, L.; Feng, X. ACS Catal. 2016, 6, 2482.

[6] Bang, J.; Oh, C.; Lee, E.; Jeong H.; Lee, J.; Ryu, J. Y.; Kim, J.; Yu, C.-M. Org. Lett. 2018, 20, 1521.

[7] Zhang, H.-J.; Yin, L. J. Am. Chem. Soc. 2018, 140, 12270.

[8] (a) Zhang, H.-J.; Shi, C.-Y.; Zhong, F.; Yin, L. J. Am. Chem. Soc. 2017, 139, 2196.

(b) Zhong, F.; Yue, W.-J.; Zhang, H.-J.; Zhang, C.-Y.; Yin, L. J. Am. Chem. Soc. 2018, 140, 15170.

[9] Zhong, F.; Xue, Q.-Y.; Yin, L. Angew. Chem., Int. Ed. 2020, 59, 1562.

(Lu, Y.) 